\title{
Relação entre a mobilidade da articulação talocrural e a úlcera venosa
}

\author{
Relationship between talocrural joint mobility and venous ulcer \\ Cleusa Ema Quilici Belczak¹, Gildo Cavalheri Jr. ${ }^{2}$, José Maria Pereira de Godoy ${ }^{3}$, \\ Roberto Augusto Caffaro ${ }^{4}$, Sergio Quilici Belczak ${ }^{5}$
}

\section{Resumo}

Contexto: O presente estudo avalia a mobilidade da articulação talocrural nos seis estágios clínicos da classificação CEAP (clínica, etiológica, anatômica e patofisiológica do International Consensus Committee Reporting Standards on Venous Disease) para doença venosa utilizando a goniometria, e detecta redução da mobilidade articular nos estágios mais avançados da doença, C5 e C6 (úlcera cicatrizada ou ativa).

Objetivo: Investigar a existência de uma relação entre a severidade clínica da doença venosa crônica dos membros inferiores e a diminuição do grau de mobilidade da articulação talocrural.

Método: Selecionaram-se aleatoriamente 120 membros pertencentes a 88 pacientes brancas, que foram separados com base em sua apresentação clínica de acordo com a categoria $C$ da classificação CEAP, sendo distribuídos em 6 grupos pertencentes às categorias de $\mathrm{C} 0-\mathrm{C} 1$ (grupo controle) até C6, com 20 membros cada um e médias de idade próximas para cada grupo. $\mathrm{O}$ grau de mobilidade do tornozelo foi acessado por goniometria de apoio plantar em posição de decúbito supino.

Resultados: Os grupos C de CEAP apresentam diferença significativa em relação ao grau de mobilidade da articulação talocrural medida por goniometria $(p<0,001)$. C6 difere significativamente dos demais grupos $(\mathrm{p}<0,05)$; C5 difere significativamente de $\mathrm{C} 6, \mathrm{C} 3, \mathrm{C} 2 \mathrm{e} \mathrm{C} 0-\mathrm{C} 1(\mathrm{p}<0,05)$, mas não apresenta diferença significativa do grupo $\mathrm{C} 4$; $\mathrm{C} 4$ difere significativamente do grupo C6 $(\mathrm{p}<0,05)$ e não difere dos demais grupos; $\mathrm{C} 0-\mathrm{C} 1, \mathrm{C} 2 \mathrm{e} \mathrm{C} 3$ não apresentam diferença significativa entre si e em relação a $\mathrm{C} 4$, e diferem dos grupos C5 e C6 $(\mathrm{p}<0,05)$. O nível de significância utilizado para os testes foi de $5 \%$.

Conclusão: Existe relação entre a severidade clínica da insuficiência venosa crônica dos membros inferiores e a diminuição do grau de mobilidade da articulação talocrural, e ela se faz mais evidente na presença de úlcera venosa ativa ou cicatrizada.

Palavras-chave: Insuficiência venosa, articulação talocrural, úlcera venosa.

\begin{abstract}
Background: This study assesses talocrural joint mobility considering the six stages of CEAP classification (clinical, etiologic, anatomic and pathophysiologic by the International Consensus Committee reporting standards on venous disease) for venous disease using goniometry, and detects reduction in joint mobility in more advanced stages of the disease, C5 and C6 (healed or active ulcer).
\end{abstract}

Objective: Investigate the existence of a relationship between clinical severity of chronic venous disease of the lower limbs and reduction in talocrural joint mobility.

Methods: A total of 120 limbs from 88 Caucasian patients were randomly selected. They were divided based on clinical presentation according to the $\mathrm{C}$ clinical category of CEAP, being distributed into six groups belonging to categories from $\mathrm{C} 0-\mathrm{C} 1$ (control group) to $\mathrm{C} 6$, with 20 limbs each and similar mean age for each group. Range of ankle mobility was assessed by goniometry in the supine position.

Results: $\mathrm{C}$ groups on the CEAP classification showed significant difference in relation to talocrural joint mobility measured by goniometry $(p<0.001)$. C6 was significantly different from the other groups ( $\mathrm{p}<0.05)$; C5 was significantly different from C6, C3, C2 and $\mathrm{C} 0-\mathrm{Cl}(\mathrm{p}<0.05)$, but with no significant difference from $\mathrm{C} 4$; $\mathrm{C} 4$ was significantly different from $\mathrm{C} 6(\mathrm{p}<0.05)$ and not different from the other groups; $\mathrm{C} 0-\mathrm{C} 1, \mathrm{C} 2$ and $\mathrm{C} 3$ were not significantly different between themselves and in relation to $\mathrm{C} 4$, but were different from $\mathrm{C} 5$ and C6 ( $p<0.05)$. Significance level used for tests was 5\%.

Conclusion: There is a relationship between clinical severity of chronic venous insufficiency of the lower limbs and reduction in talocrural joint mobility, which is more evident in the presence of active or healed venous ulcer.

Keywords: Venous insufficiency, talocrural joint, venous ulcer.

1. Doutoranda em Cirurgia Geral, Faculdade de Ciências Médicas, Santa Casa de São Paulo, São Paulo, SP. Professora, Curso de PósGraduação em Reabilitação Linfovenosa, Faculdade de Medicina de São José do Rio Preto (FAMERP), São José do Rio Preto, SP.

2. Fisioterapeuta. Mestre em Ciência da Saúde, FAMERP, São José do Rio Preto, SP.

3. Doutor, livre-docente. Professor adjunto, Cardiologia e Cirurgia Cardiovascular, Departamento de Cirurgia, FAMERP, São José do Rio Preto, SP. Pesquisador, Conselho Nacional de Desenvolvimento Científico e Tecnológico (CNPq).

4. Doutor. Professor adjunto, Disciplina de Cirurgia Vascular, Departamento de Cirurgia, Faculdade de Ciências Médicas, Santa Casa de São Paulo, São Paulo, SP.

5. Residente, Cirurgia Vascular, Faculdade de Medicina, Universidade de São Paulo (USP), São Paulo, SP.

Artigo submetido em 11.12.06, aceito em 17.05.07.

J Vasc Bras 2007;6(2):149-155.

Copyright $\odot 2007$ by Sociedade Brasileira de Angiologia e de Cirurgia Vascular 


\section{Introdução}

Cada vez mais a insuficiência venosa crônica (IVC) dos membros inferiores (MMII), além de ser causa de incapacidade e constrangimento do indivíduo acometido, representa importante problema de ordem socioeconômica na civilização contemporânea. Em suas formas mais graves, como na úlcera de estase venosa, última expressão desta enfermidade, pode conduzir à invalidez e ser motivo de elevados custos aos cofres públicos. A avaliação abrangente e o manejo terapêutico adequado destes pacientes encontra-se ainda aquém do esperado. Assim, seu aprimoramento constitui uma grande necessidade na prática angiológica cotidiana.

Sabe-se que a hemodinâmica do retorno venoso dos MMII é complexa, mal compreendida e multifatorial ${ }^{1}$. A elevação da pressão venosa ambulatorial (PVA) no flebopata crônico tem sido bem documentada ${ }^{2-4}$; no entanto, outros parâmetros pertinentes à deterioração da hemodinâmica venosa não têm sido devidamente valorizados, sendo pouco estudados ou citados. As patologias venosas ocorrem em conseqüência à obstrução e/ou refluxo com ou sem disfunção da bomba muscular da panturrilha (BMP) $)^{5,6}$ que, como se sabe, sofre influência direta da mobilidade talocrural ${ }^{7-9}$. A deficiência da BMP em membros com úlcera ativa é fator determinante da gravidade do prognóstico.

O objetivo deste trabalho é investigar a existência de uma relação linear entre a presença da úlcera de estase venosa e a limitação de mobilidade da articulação talocrural medida por goniometria.

\section{Métodos}

Foram avaliados aleatoriamente 120 membros pertencentes a 88 mulheres adultas brancas que buscaram tratamento para IVC dos MMII no Centro Vascular João Belczak, de Maringá (PR, Brasil). Os membros pertenciam indiferentemente ao lado esquerdo ou direito, foram selecionados clinicamente e classificados de acordo com consenso protocolado do Comitê Internacional em Doença Venosa, denominado classificação
$\mathrm{CEAP}($ no qual $\mathrm{C}=$ clínica, $\mathrm{E}=$ etiologia, $\mathrm{A}=$ segmento anatômico e $\mathrm{P}=$ fisiopatologia), perfazendo 6 grupos com 20 membros cada um, variando desde $\mathrm{C} 0-\mathrm{C} 1$ até $\mathrm{C} 6$, onde $\mathrm{C} 0-\mathrm{C} 1$ será considerado como grupo controle, pois equivale à ausência de sinais e sintomas ou à presença somente de telangiectasias; $\mathrm{C} 2$ veias varicosas; C3 edema, C4 alterações na pele (pigmentação, eczema varicoso, lipodermatoesclerose); C5 úlcera cicatrizada e C6 úlcera ativa ${ }^{10}$. Todas as participantes faziam parte do mesmo grupo populacional urbano e assinaram um termo de consentimento após terem sido informadas sobre o objetivo deste estudo, que foi previamente submetido à apreciação e posterior aprovação por comitê de ética da universidade privada CESUMAR.

Foram excluídos fatores como a presença de indivíduos do sexo masculino ou de outro grupo racial que não a raça branca e também aqueles que se encontravam em idades extremas. Excluíram-se pacientes isquêmicas, hipertensas, diabéticas ${ }^{11}$ e /ou aquelas que estavam sob uso de medicamentos que poderiam interferir na formação de edema, tais como diuréticos e hormônios, e portadoras de edema dos membros inferiores de origem sistêmica como insuficiência cardíaca congestiva, renal, hepática, mixedema e doenças articulares traumáticas ou reumáticas.

Após terem sido consultadas, as pacientes foram submetidas à goniometria da articulação talocrural, denominada na literatura anglo-saxônica pela sigla ROAM (range of ankle motion) que utilizaremos neste artigo, cujo valor equivale à soma da flexão plantar mais a dorsal. A medida foi executada em posição de decúbito dorsal, sempre pelo mesmo fisioterapeuta do serviço $^{12}$, em uma única medida no período da manhã, com o auxílio de goniômetro portátil de apoio plantar (que impede a interferência das outras articulações do pé), e está constituído por duas lâminas metálicas articuladas (Figura 1), com uma folha de papel interposta onde se desenhou o ângulo obtido e se calculou o valor com um simples transferidor. 


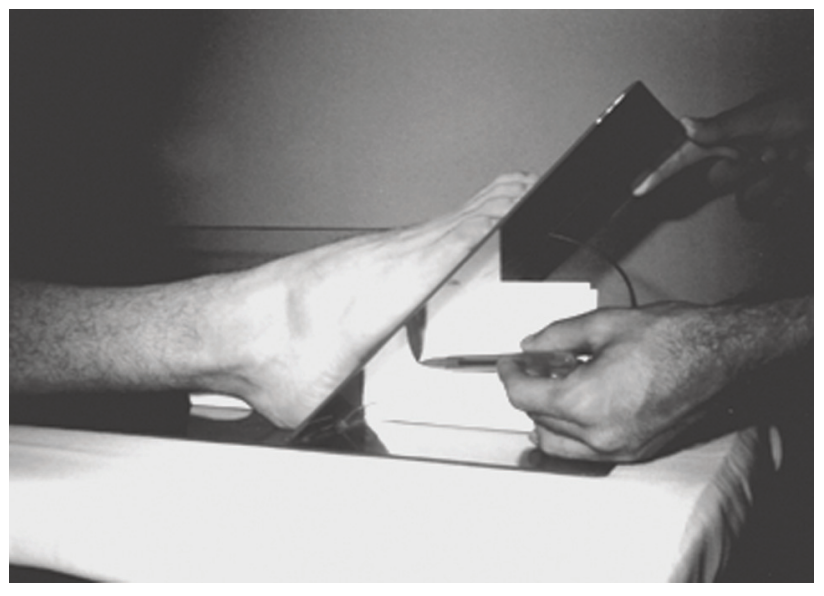

Figura 1 - Goniômetro de apoio plantar Belczak 1 para medida da mobilidade da articulação talocrural sem influência de outras articulações do pé

\section{Resultados}

Podemos observar, pela tabela acima, que os grupos de CEAP apresentam diferença significativa em relação ao grau de mobilidade da articulação talocrural medida por goniometria $(\mathrm{p}<0,001)$.

Por meio do teste de comparação múltipla de Bonferroni, observamos que o grupo C6 difere significativamente dos demais grupos $(\mathrm{p}<0,05)$. O grupo C5 difere significativamente dos grupos C6, C3, C2 e C0-C1 $(\mathrm{p}<0,05)$, mas não apresenta diferença significativa do grupo C4 que difere significativamente do grupo C6 $(\mathrm{p}<0,05)$ e não difere dos demais grupos. Os grupos $\mathrm{C} 0-\mathrm{C} 1, \mathrm{C} 2$ e C3 não apresentam diferença significativa entre si e em relação a C4 e diferem dos grupos C5 e C6 $(\mathrm{p}<0,05)$ (Figura 2).

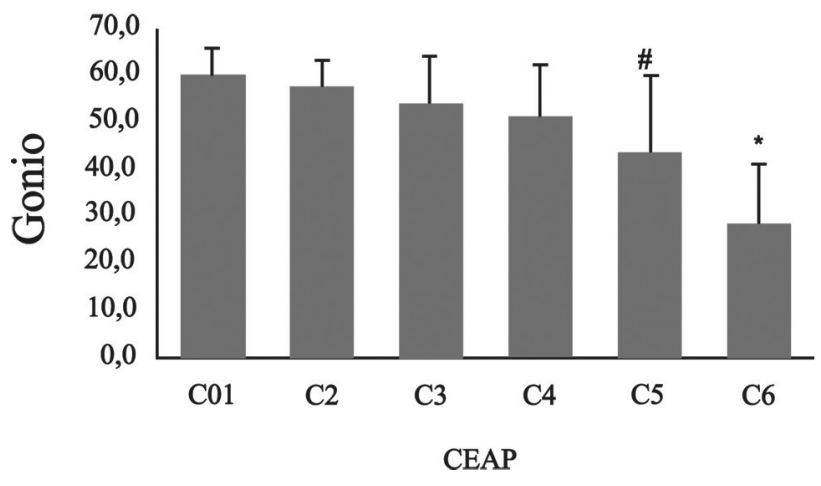

* p < 0,05 em relação a C0-C1, C2, C3, C4 e C5; (\#) p <0,05 em relação a C0-C1, C2, C3. Comparação dos grupos separados pela classificação CEAP em relação à idade.

Figura 2 - Médias e desvio padrão dos valores obtidos pela goniometria de apoio plantar da articulação talocrural segundo o grupo da classificação CEAP

Podemos observar, pela Tabela 3 e Figura 3, que os grupos caracterizados pela classificação CEAP não apresentam diferença significativa em relação à idade $(\mathrm{p}=0,559)$.

Tabela 1 - Comparação dos pacientes agrupados pela classificação CEAP em relação à medida do grau de mobilidade da articulação talocrural avaliada por goniometria e a idade

\begin{tabular}{lcccc}
\hline Grupos CEAP & Média goniométrica articulação talocrural & DP & Idade das pacientes & DP \\
\hline C0- C1 & 60,15 & 5,57 & 55,55 & 7,69 \\
C2 & 57,40 & 6,24 & 58,35 & 7,77 \\
C3 & 54,15 & 9,79 & 58,05 & 11,87 \\
C4 & 51,35 & 10,73 & 57,20 & 10,76 \\
C5 & 43,50 & 16,60 & 59,65 & 8,92 \\
C6 & 28,35 & 12,81 & 60,90 & 8,57 \\
\hline
\end{tabular}

CEAP = classificação clínica, etiológica, anatômica e patofisiológica; $\mathrm{DP}=$ desvio padrão. 


\section{Análise estatística}

Inicialmente, todas as variáveis foram analisadas descritivamente. Para as variáveis quantitativas, esta análise foi feita por meio da observação dos valores mínimos e máximos e do cálculo de médias, desvio padrão e medianas. Para a análise da hipótese de igualdade das médias entre os grupos, utilizou-se a análise de variância a um fator com comparações múltiplas por meio do teste de Bonferroni ${ }^{13}$. O nível de significância utilizado para os testes foi de 5\%.

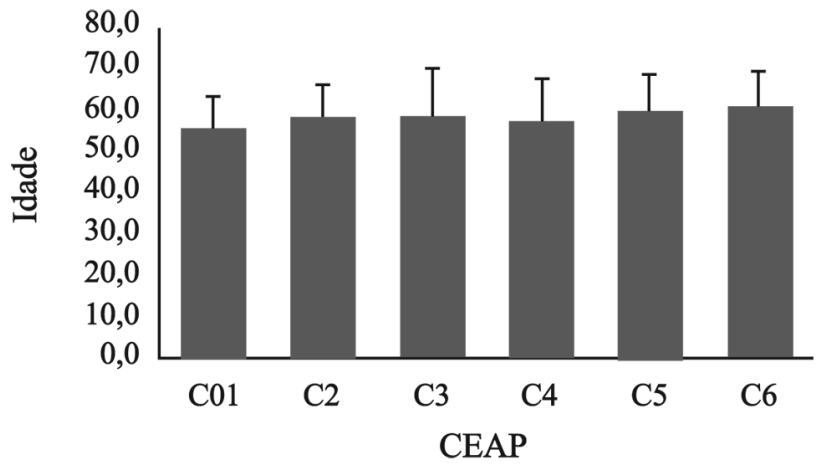

Figura 3 - Médias e desvio padrão dos valores de idade, segundo o grupo da classificação CEAP

Tabela 2 - Valores de média, desvio padrão (DP), mediana, mínimo e máximo dos valores de goniometria da articulação talocrural, segundo o grupo de CEAP

\begin{tabular}{lccccccc}
\hline CEAP & $\mathbf{n}$ & Média & DP & Mediana & Mínimo & Máximo & $\mathbf{p}^{*}$ \\
\hline C0- C1 & 20 & 60,15 & 5,57 & 60,50 & 50,00 & 73,00 & \\
C2 & 20 & 57,40 & 6,24 & 58,50 & 48,00 & 67,00 & \\
C3 & 20 & 54,15 & 9,79 & 56,50 & 27,00 & 68,00 & $<0,001$ \\
C4 & 20 & 51,35 & 10,73 & 53,00 & 29,00 & 70,00 & \\
C5 & 20 & 43,50 & 16,60 & 48,00 & 8,00 & 68,00 & \\
C6 & 20 & 28,35 & 12,81 & 25,00 & 5,00 & 55,00 & \\
\hline
\end{tabular}

$\mathrm{CEAP}=$ classificação clínica, etiológica, anatômica e patofisiológica; $\mathrm{DP}=$ desvio padrão.

* Nível descritivo de probabilidade da análise de variância a um fator.

Tabela 3 - Valores de média, desvio padrão, mediana, mínimo e máximo dos valores de idade, segundo o grupo de CEAP

\begin{tabular}{lccccccc}
\hline CEAP & $\mathbf{n}$ & Média & DP & Mediana & Mínimo & Máximo & p* \\
\hline C0-C1 & 20 & 55,55 & 7,69 & 56,50 & 36,00 & 69,00 & \\
C2 & 20 & 58,35 & 7,77 & 58,00 & 47,00 & 71,00 & \\
C3 & 20 & 58,05 & 11,87 & 57,50 & 39,00 & 77,00 & 0,559 \\
C4 & 20 & 57,20 & 10,76 & 59,50 & 39,00 & 75,00 & \\
C5 & 20 & 59,65 & 8,92 & 59,50 & 43,00 & 78,00 & \\
C6 & 20 & 60,90 & 8,57 & 62,00 & 45,00 & 75,00 & \\
\hline
\end{tabular}

* Nível descritivo de probabilidade da análise de variância a um fator. 


\section{Discussão}

A mobilidade da articulação talocrural aliada à competência valvular venosa trabalhando simultaneamente, constitui o motor que impulsiona o retorno do sangue venoso ao coração, via as relações anatômicas existentes entre nervos, músculos, tendões, cápsula, ligamentos e cartilagens ${ }^{7}$. Tem sido observado que a redução do ROAM constitui fator agravante da IVC dos MMII, pois a anquilose total e permanente do tornozelo torna os pacientes incuráveis ao limitar ou mesmo anular a ação da mais importante bomba impulsoaspirativa dos membros inferiores, que é a bomba muscular da panturrilha ${ }^{14}$.

Loettzke, em $1956^{15}$, descreveu que a camada profunda da fáscia crural relaciona-se intimamente com o tendão de Aquiles que, durante a marcha, é tracionado para trás pela flexão plantar e depois para frente pela flexão dorsal, definindo que esta tração promove a contração do músculo tríceps-sural, cuja ativação completa só se realiza mediante movimentação articular adequada $^{16}$.

Foram Krämer \& Gudat ${ }^{16}$ que primeiro introduziram o ângulo talocrural para prover a medida exata da mobilidade do tornozelo, utilizando-a não só para ampliar o conhecimento da fisiopatologia da IVC e de outras enfermidades como também para programar terapias mais abrangentes.

Schmeller, em $1990^{17}$, demonstrou que se o movimento talocrural ou tíbio-társico for inibido durante a marcha pela presença de saltos altos ou pelo enrijecimento articular, seja qual for sua etiologia, a eficiência da "bomba do tornozelo", como chamada por Bolliger $\&$ Borgnis em $1970^{18}$, fica comprometida e, conseqüentemente, o retorno venoso.

Segundo os estudos de Roaas \& Andersson ${ }^{19}$, homens adultos normais em posição de decúbito dorsal apresentam em média um total de 55 a 66 graus de ROAM. Notaram não existir diferença estatisticamente significativa entre a mobilidade do tornozelo do lado esquerdo ou direito. Assim, em nossa pesquisa, selecionamos os membros indiferentemente quanto ao lado.
Oppel, na Alemanha, em $1987^{20}$, além de encontrar diferenças entre os grupos raciais branco e amarelo (alemães e japoneses), constatou uma mobilidade maior nas mulheres que nos homens e notou que, com o avançar dos anos, esta diferença não ocorria, dados confirmados por Nigg et al. em $1992^{21}$. Grimston et al. ${ }^{22}$ comprovaram que o ROAM é $15 \%$ menor na faixa etária da oitava década quando comparado ao da terceira. Em ambos os sexos, as forças musculares ativas responsáveis pelos movimentos, decrescem conforme aumenta a idade, pois os ligamentos e o aparelho capsular enrijecem, e a cartilagem se modifica, reduzindo a movimentação. Baseados nestes achados, procuramos excluir de nossa pesquisa indivíduos do sexo masculino, mulheres com idades extremas (Tabela 2) e/ou que pertencessem a outro grupo racial que não o branco, características que poderiam interferir nos resultados.

A associação de úlcera venosa, complicação mais grave da IVC dos MMII, com a limitação da articulação talocrural, foi observada pela primeira vez em 1931 por Dickson Wright ${ }^{23}$. Depois, Ruckley ${ }^{24}$ demonstrou, em 1982, em estudo sobre 827 pacientes com úlcera, que $32 \%$ tinham limitação severa do movimento do tornozelo, nos quais apenas 9\% apresentavam artrite reumatóide. Na nossa amostra, constatou-se a menor mobilidade exatamente em C6 (úlcera ativa).

Segundo Tiernay et al. ${ }^{25}$, uma disfunção venosa importante está presente apenas cinco dias após uma fratura aguda estabilizada do tornozelo e leva 18 semanas para se recuperar. Timi et al. ${ }^{26}$, realizaram estudo comparativo da mobilidade talocrural medida por goniometria de apoio plantar em três grupos de pacientes classificados pela CEAP em C0, C3 e C4, perfazendo um total de 120 membros, e encontraram, ao comparar C4 com C3, uma diferença de 36\% entre as médias obtidas, e entre $\mathrm{C} 3$ com o controle $\mathrm{C} 0$, apenas $11 \%$. Dessa forma, concluíram que pacientes classificados como C4 estão associados a um ROAM menor que os pacientes $\mathrm{C} 3$ e C0. Deduziram que a diferença entre os pacientes residia especialmente na presença de lesões tróficas. Em nossa amostra, os pacientes C4 (aqueles 
com lesão trófica), apenas diferiam estatisticamente dos C6 e não dos outros grupos.

Christopoulos et al., em $1989^{5}$, pesquisando a etiologia da úlcera de estase venosa, salientaram a responsabilidade da BMP no processo, ao constatar que com uma boa fração de ejeção medida pela pletismografia a ar, mesmo frente a refluxos importantes, não ocorria a formação de úlcera, enquanto que com pequenos refluxos e fração de ejeção baixa, esta complicação podia existir.

Back et al., em 19957, enfatizaram que a anquilose talocrural se inicia "antes" do aparecimento da úlcera e constataram que os membros com IVC têm um ROAM que diminui conforme aumenta a gravidade dos sintomas clínicos da doença, sendo que aqueles com úlcera ativa ou cicatrizada, apresentam uma mobilidade significativamente menor do que aqueles sem úlcera, o que também foi encontrado por nós.

Dix et al. ${ }^{8}$ constataram que membros com todo tipo de doença venosa, desde simples varicosidades até ulceração, estão associados a hipertensão venosa e limitação talocrural. Em sua amostra, até mesmo os pacientes $\mathrm{C} 2$ apresentaram flexibilidade do tornozelo reduzida, o que difere de nosso grupo.

A redução de ROAM observada conforme aumenta o grau de severidade clínica sugere que a hipertensão venosa seja uma das causas desta limitação. Vale lembrar que a partir de C3 existe edema e que seguramente este fato também contribui para a diminuição da flexibilidade articular do tornozelo, instalando-se assim um ciclo vicioso.

Um fator limitante de nossa pesquisa deve-se à medida do ROAM ter sido realizada em decúbito dorsal. O ideal seria executá-la em deambulação com o peso do corpo atuando para se obter uma avaliação com a função mais próxima do normal. Por outro lado, o fato de pacientes com C5 e C6 normalmente usarem elastocompressão a longo prazo pode também ser fator determinante de uma ligeira atrofia no tornozelo. $\mathrm{Na}$ IVC dos MMII pode ocorrer dor intensa que, por sua vez, pode causar rigidez articular de graus variados e até irreversíveis (Figura 4), promovendo um encurtamento secundário do tendão de Aquiles que resulta em uma deformidade em flexão plantar fixa, que pode ensombrecer o prognóstico e colaborar para o ciclo vicioso ${ }^{27}$.

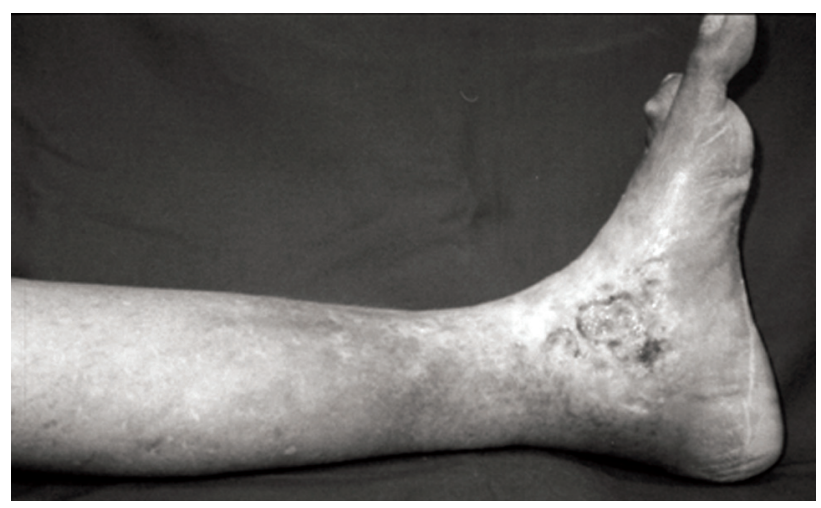

Figura 4 - Paciente C6, pós trombótica, portadora de úlcera de estase venosa de longa duração com limitação importante da mobilidade da articulação talocrural. $\mathrm{ROAM}=8^{\circ}$

Frente à importância do tema, aos dados obtidos e considerando-se a literatura consultada, sugere-se que se agregue ao exame físico do flebopata grave a realização da goniometria do tornozelo. É um método simples, barato e repetível, que pode identificar de forma o mais precoce possível o comprometimento da mobilidade talocrural. Considerando-se que toda terapia deva se orientar pelos fenômenos fisiopatológicos que dão origem ao problema, este dado poderá ser de grande utilidade na programação de um tratamento clínicofísico-reabilitador. Indiretamente, ao se ampliar o ROAM, se melhorará a função da $\mathrm{BMP}^{28-30} \mathrm{e}$, conseqüentemente, serão minimizadas as repercussões nefastas da IVC constituída, evitando a evolução mórbida do processo e melhorando a qualidade de vida do indivíduo.

\section{Conclusão}

A IVC dos membros inferiores está associada a uma redução da mobilidade da articulação talocrural, que pode ser identificada pela goniometria. Esta relação se intensifica e se torna mais evidente conforme aumenta o grau de severidade clínica da própria enfermidade com a presença de úlcera venosa ativa ou cicatrizada. 


\section{Referências}

1. Welkie JF, Comerota AJ, Katz ML, Aldridge SC, Kerr RP, White JV. Hemodynamic deterioration in chronic venous disease. J Vasc Surg. 1992;16:733-40.

2. Barber RF, Shatara FI. The varicose disease. NY State J Med. 1925;31:574-80.

3. Pollack AA, Wood EH. The venous pressure in the saphenous vein at the ankle in man, during exercise and changes in posture. J Appl Physiol. 1949;1:649-62.

4. Nicolaides AN, Hussein MK, Szendro G, Christopoulos D, Vasdekis S, Clarke H. The relation of venous ulceration with ambulatory venous pressure measurements. J Vasc Surg. 1993;17:414-9.

5. Christopoulos D, Nicolaides AN, Cook A, Irvine A, Galloway JM, Wilkinson A. Pathogenesis of venous ulceration in relation to the calf muscle pump function. Surgery. 1989;106:829-35.

6. Araki CT, Back TL, Padberg FT, et al. The significance of calf muscle pump function in venous ulceration. J Vasc Surg. 1994;20:872-7; discussion 878-9.

7. Back TL, Padberg FT Jr., Araki CT, Thompson PN, Hobson RW 2nd. Limited range of motion of the ankle joint is a significant factor in venous ulceration. J Vasc Surg. 1995;22:519-23.

8. Dix FP, Brooke R, McCollum CN. Venous disease is associated with an impaired range of ankle movement. Eur J Vasc Endovasc Surg. 2003;25:556-61.

9. Orsted HL, Radke L, Gorst R. The impact of musculoskeletal changes on the dynamics of the calf muscle pump. Ostomy Wound Manage. 2001;47:18-24.

10. Porter JM, Moneta GL. Reporting standards in venous disease: an update. International Consensus Committee on Chronic Venous Disease. J Vasc Surg. 1995;21:635-45.

11. Tinley P, Taranto M. Clinical and dynamic range of ankle motion techniques in subjects with and without diabetes mellitus. J Am Podiatr Med Assoc. 2002;92:136-42.

12. Somers DL, Hanson JA, Kedzierski CM, Nestor KL, Quinlivan KY. The influence of experience on the reliability of goniometric and visual measurement of forefoot position. J Orthop Sports Phys Ther. 1997;25:192-202.

13. Rosner B. Fundamentals of biostatistics. 2nd ed. Boston: PWS Publishers; 1986.

14. Belczak Neto J, Belczak CEQ. A importância da goniometria do tornozelo na insuficiência venosa crônica dos membros inferiores. In: Thomaz JB, Belczak CEQ, editores. Tratado de flebologia e linfologia. Rio de Janeiro: Rubio; 2006. p. 459-68.

15. Loetzke HH. Über die Achlles-sehne mit ihren Faszienverhältnissen beim Menschen und den Subcutanvarizen im Bereich der Wadenmuskulatur. Anat Anz. 1956;103:287-304.

16. Kramer J, Gudat W. Der Talokrural-Winkel. Z Orthop Ihre Grenzgeb. 1980;118;855-8.
17. Schmeller W, Steidel G, Borgis KJ. Über den bewegungsumfang im oberen sprunggelenk bei venengesunden und venenkraken: ein beitrag zum arthrogenen staunngssyndrom. Phlebol Proktol. 1990;19:100-10.

18. Bolliger AA, Borgnis FE. Ein verbesserter Ultraschallströmungsmesser für intravasale Messungen. In: May R. Mefmethoden in der Venenchirurgie. Bern: Huber; 1971.P.137-43.

19. Roaas A, Andersson GB. Normal range of motion of the hip, knee and ankle joints in male subjects, 30-40 years of age. Acta Orthop Scand. 1982;53:205-8.

20. Oppel U, Higushi F. Talokrural-Winkel und Sprunggelenksbeweglichkeit bei Deutschen und Japanern. Z Orthop. 1987;125:243-6.

21. Nigg BM, Fisher V, Allinger TL, Ronsky JR, Engsberg JR. Range of motion of the foot as a function of age. Foot Ankle. 1992;13:336-43.

22. Grimston SK, Nigg BM, Hanley DA, Engsberg JR. Differences in ankle joint complex range of motion as a function of age. Foot Ankle. 1993;14:215-22.

23. Wright AD. The treatment of indolent ulcer of the leg. Lancet. 1931;1:457-60.

24. Ruckley CV, Dale JJ, Callam MJ, Harper DR. Causes of chronic leg ulcer. Lancet. 1982;2:615-6.

25. Tierney S, Burke P, Fitzgerald P, O'Sullivan T, Grace P, Bouchier-Hayes D. Ankle fracture is associated with prolonged venous dysfunction. Br J Surg. 1993;80:36-8.

26. Timi JR, Futigami AY, Belczak SQ, Pradella FM. Ankle ankylosis and its importance in chronic venous disease. Int Angiol. 2005;24:148.

27. Browse NL, Burnand KG, Thomas ML. Síndrome da falência contrátil da panturrilha. In: Browse NL, Burnand KG, Thomas ML. Doenças venosas. 2 ed. Rio de Janeiro: DiLivros; 2001. p. 433-60.

28. Kan YM, Delis KT. Hemodynamic effects of supervised calf muscle exercise in patients with venous leg ulceration: a prospective controlled study. Arch Surg. 2001;136:1364-9.

29. Yang D, Vandongen YK, Stacey MC. Effect of exercise on calf muscle pump function in patients with chronic venous disease. Br J Surg. 1999;86:338-41.

30. Belczak Neto J, Belczak CEQ. Reabilitação cinesiofisiátrica do flebopata crônico. In: Thomaz JB, Belczak CEQ, editores. Tratado de flebologia e linfologia. Rio de Janeiro: Rubio; 2006. p. $460-84$.

Correspondência:

Cleusa Ema Quilici Belczak

Centro Vascular João Belczak

Av. Tiradentes, 1081

CEP 87013-260 - Maringá, PR

E-mail: cleusabelczak@yahoo.com.br 